\title{
A REPORT ON PHASE 2 OF THE FIFTH INTERNATIONAL RADIOCARBON INTER- COMPARISON (VIRI)
}

\author{
E Marian $\operatorname{Scott}^{1} \bullet$ Gordon T Cook ${ }^{2}$ Philip Naysmith ${ }^{2}$
}

ABSTRACT. The Fifth International Radiocarbon Intercomparison (VIRI) continues the tradition of the TIRI (third) and FIRI (fourth) (Scott 2003) intercomparisons and operates in addition to any within-laboratory quality assurance measures as an independent check on laboratory procedures. VIRI is a phased intercomparison; results for the first phase, which employed grain samples, were reported in Scott et al. (2007). The second phase, involving bone samples, is reported here. The third and final phase, which includes samples of peat, wood, and shell, has also been completed and a companion paper appears in these proceedings.

Five bone samples were made available and included Sample E: mammoth bone ( $>5$ half-lives); Sample F: horse bone (from Siberia, excavated in 2001; and Samples H and I: whale bones (approximately 2 half-lives). Sample G (human bone) was accessible only to accelerator mass spectrometry (AMS) laboratories because of the limited amount of sample available. More than 40 laboratories participated in Phase 2 and consensus values for the ages were as follows: Sample $\mathrm{E}=39,305{ }^{14} \mathrm{C}$ yr $\mathrm{BP}$ (standard deviation $[1 \sigma]=121 \mathrm{yr})$; Sample F = $2513 \mathrm{yr} \mathrm{BP}(1 \sigma=5 \mathrm{yr})$; Sample $\mathrm{G}=969 \mathrm{yr} \mathrm{BP}(1 \sigma=5 \mathrm{yr})$; Sample $\mathrm{H}=9528$ yr BP $(1 \sigma=7 \mathrm{yr})$; and Sample $\mathrm{I}=8331 \mathrm{yr}$ BP $(1 \sigma=6 \mathrm{yr})$. Sample $\mathrm{G}$ had previously been dated by 4 laboratories and a weighted mean of $934 \pm 12 \mathrm{yr}$ BP had been quoted. Sample I had previously been dated at $8335 \pm 25 \mathrm{yr}$ BP and Sample H had been dated at $9565 \pm 130 \mathrm{yr}$ BP. Results for Sample H and Sample I are in good agreement with the previous results; Sample $\mathrm{G}$ results, however, give a value that is significantly older than the previously reported results.

\section{INTRODUCTION}

The Fifth International Radiocarbon Intercomparison (VIRI) has continued the tradition of the TIRI (third) and FIRI (fourth) intercomparisons (Scott 2003) as a ${ }^{14} \mathrm{C}$ community project, with samples provided by participants and a substantial participation rate. Over the 4-yr lifetime of VIRI, the first suite of samples (grain) was sent out in September 2004. The second suite of samples comprised bone and was distributed in October 2005, with results returned by late 2006. The final suite of samples included a wide variety of materials and was completed in 2008.

Not all laboratories that had previously participated in Phase 1 participated in Phase 2, since bone is not a routinely measured sample in all laboratories. A total of 42 laboratories, identified in Table 1, reported results. As always, the actual number of results submitted was greater than the number of laboratories since several laboratories submitted results using several independent systems. As a consequence, more than 60 sets of results were returned. One of the difficulties reported, especially for the radiometric laboratories, was that the sample size did not meet their routine requirements. For radiometric laboratories, we had distributed samples ranging from $60-100 \mathrm{~g}$. A few laboratories also reported difficulty with the sample pretreatment (specifically, collagen extraction).

\section{Sample Descriptions}

Sample E: Mammoth Bone (>5 half-lives)

This bone is from a site called Quartz Creek, Dawson City, Yukon Territory. The bone is a portion of the pelvis of a Mammuthus sp. specimen. The sample was collected in August 2003 by Ross Barnett of the Zoology Department, University of Oxford. It was supplied by Tom Higham of ORAU. In an initial test of the material, $0.58 \mathrm{~g}$ of collagen was recovered from $5 \mathrm{~g}$ of bone. The $\%$ carbon of this collagen sample was $41 \%$.

\footnotetext{
${ }^{1}$ Department of Statistics, University of Glasgow, Glasgow G12 8QW, Scotland. Corresponding author. Email: marian@stats.gla.ac.uk.

${ }^{2}$ SUERC, Scottish Enterprise Technology Park, East Kilbride G75 0QF, Scotland.
}

C 2010 by the Arizona Board of Regents on behalf of the University of Arizona Proceedings of the 20th International Radiocarbon Conference, edited by A J T Jull RADIOCARBON, Vol 52, Nr 2-3, 2010, p 846-858 
Table 1 Participating laboratories.

\begin{tabular}{|c|c|c|}
\hline Lab name & Lab method ${ }^{\mathrm{a}}$ & Country \\
\hline Laboratorio de Tritio y Radiocarbone, La Plata & LSC & Argentina \\
\hline ANSTO & AMS & Australia \\
\hline VERA, University of Vienna & AMS & Austria \\
\hline Belarus Academy of Science & LSC & Belarus \\
\hline Royal Institute for Cultural Heritage & AMS & Belgium \\
\hline Aarhus AMS Dating Laboratory & AMS & Denmark \\
\hline Dating Laboratory, University of Helsinki & AMS & Finland \\
\hline Centre de Datation par le Radiocarbone, Lyon & AMS, LSC & France \\
\hline Heidelberg Akademie der Weissenschaften & GPC & Germany \\
\hline Radiocarbon Laboratory, Cologne & GPC & Germany \\
\hline AMS Laboratory, Erlangen & AMS & Germany \\
\hline Leibniz Institute for Applied Geosciences, Hannover & GPC & Germany \\
\hline Leibniz Labor, Kiel & AMS & Germany \\
\hline Laboratory of Environmental Studies of INR/HAS & GPC & Hungary \\
\hline CEDAD, University of Lecce & AMS & Italy \\
\hline ENEA & LSC & Italy \\
\hline CIRCE, 2nd University Naples & AMS & Italy \\
\hline Weizmann Institute & AMS & Israel \\
\hline Centre for Chronological Research, Nagoya University & AMS & Japan \\
\hline University Museum, University of Tokyo & AMS & Japan \\
\hline Universiteit Utrecht & AMS & Netherlands \\
\hline Centre for Isotope Study, Groningen & AMS, GPC & Netherlands \\
\hline Rafter Radiocarbon Laboratory & AMS & New Zealand \\
\hline University of Waikato & AMS, LSC & New Zealand \\
\hline Radiocarbon Lab, Trondheim & AMS & Norway \\
\hline Poznań Radiocarbon Laboratory & AMS & Poland \\
\hline Kraków Radiocarbon Laboratory & LSC & Poland \\
\hline Gliwice Radiocarbon Laboratory & GPC, AMS & Poland \\
\hline Radiocarbon Laboratory, Russian Academy of Sciences & LSC & Russia \\
\hline Radiocarbon Lab, Geographical Institute, St. Petersburg & LSC & Russia \\
\hline Radiocarbon Lab, Barcelona & LSC & Spain \\
\hline Laboratorio de Datacion, Universidad de Granada & LSC & Spain \\
\hline Tandem Laboratory, Uppsala University & AMS & Sweden \\
\hline ETH/PSI, Zurich & AMS & Switzerland \\
\hline National Taiwan University & LSC & Taiwan \\
\hline Oxford Radiocarbon Accelerator lab & AMS & UK \\
\hline SUERC & AMS & UK \\
\hline Ukraine Academy of Sciences, Kiev & LSC & Ukraine \\
\hline Applied Isotope Studies, Georgia & AMS & USA \\
\hline KCCAMS, University of California & AMS & USA \\
\hline NOSAMS, WHOI & AMS & USA \\
\hline Arizona AMS facility & AMS & USA \\
\hline
\end{tabular}

${ }^{\mathrm{a}} \mathrm{AMS}=$ accelerator mass spectrometry; $\mathrm{GPC}=$ gas proportional counting; $\mathrm{LSC}=$ liquid scintillation counting.

\section{Sample F: Horse Bone ( $<1$ half-life)}

This sample was excavated in 2001 and provided by Ganna Zaitseva of the Institute of History of Material Culture, St. Petersburg, Russia. It is from an archaeological investigation of a Scythian burial site in Siberia. Some $0.34 \mathrm{~g}$ of collagen was recovered from $1.67 \mathrm{~g}$ of bone. The $\%$ carbon of this sample was $30.3 \%$. 


\section{Sample G: Human Bone}

This is a sample from a young female buried with a neonate in a waterlogged dendro-dated coffin and was provided by Alex Bayliss of English Heritage. This sample was sent to AMS laboratories only.

\section{Sample H: Whale Bone (approximately 2 half-lives)}

This whale bone sample was submitted to the University of Washington in August 1983 and is the jawbone of a whale recovered from sand deposits within a raised beach at Svalbard, Spitsbergen, Norway. It was provided by Paula Reimer of Queen's University, Belfast.

\section{Sample I: Whale Bone (approximately 2 half-lives)}

This whale bone is from the cranium of a whale (species not determined) found in August 1997 on Svalbard, and provided by Steinar Gulliksen of the National Dating Laboratory, Trondheim.

\begin{tabular}{|c|c|c|c|c|}
\hline Lab code & Lab type & Age (yr BP) & Error (yr BP) & $\delta^{13} \mathrm{C}(\%)$ \\
\hline 3 & GPC & 42,060 & 850 & -21.70 \\
\hline 4 & AMS & 37,000 & 1000 & -21.20 \\
\hline 5 & AMS & 40,700 & 1200 & -21.80 \\
\hline 5 & AMS & 40,330 & 1000 & -23.50 \\
\hline 7 & LSC & 34,150 & 1120 & -21.20 \\
\hline 8 & AMS & 39,790 & 1160 & -20.60 \\
\hline 11 & LSC & 25,530 & 500 & -21.00 \\
\hline 12 & AMS & $>41,000$ & $*$ & -21.10 \\
\hline 13 & AMS & 35,100 & 700 & -31.80 \\
\hline 13 & AMS & 42,300 & 2400 & -21.50 \\
\hline 15 & GPC & 35,550 & 600 & -22.00 \\
\hline 16 & AMS & 41,139 & 1041 & -20.80 \\
\hline 22 & AMS & 41,350 & 450 & -20.60 \\
\hline 22 & AMS & 39,950 & 500 & -20.30 \\
\hline 22 & AMS & 40,300 & 500 & -19.50 \\
\hline 22 & AMS & 40,450 & 370 & -20.10 \\
\hline 23 & AMS & 35,700 & 500 & -20.90 \\
\hline 25 & AMS & 40,320 & 760 & -20.90 \\
\hline 25 & AMS & 40,490 & 910 & -21.00 \\
\hline 26 & AMS & 41,500 & 1400 & -21.30 \\
\hline 27 & GPC & 39,950 & 410 & -20.50 \\
\hline 30 & LSC & 35,339 & 715 & -22.40 \\
\hline 31 & LSC & 35,500 & 400 & -16.80 \\
\hline 32 & LSC & 22,810 & 300 & -20.00 \\
\hline 37 & AMS & 39,000 & 300 & -20.60 \\
\hline 37 & AMS & 36,400 & 310 & -20.90 \\
\hline 38 & LSC & 24,300 & 650 & -15.80 \\
\hline 39 & AMS & 36,312 & 370 & -18.60 \\
\hline 40 & LSC & 26,550 & 700 & -21.43 \\
\hline 41 & GPC & $>42,000$ & $*$ & -20.00 \\
\hline 42 & GPC & 32,570 & 720 & -24.00 \\
\hline 43 & AMS & 40,350 & 1150 & -20.90 \\
\hline 43 & AMS & 40,100 & 1150 & -20.90 \\
\hline 44 & AMS & 40,809 & 2239 & -21.00 \\
\hline
\end{tabular}


Table 2a Results for Sample E. (Continued)

\begin{tabular}{llccl} 
Lab code & Lab type & Age $(\mathrm{yr}$ BP) & Error $(\mathrm{yr} \mathrm{BP})$ & $\delta^{13} \mathrm{C}(\%)$ \\
\hline 44 & AMS & 40,133 & 1622 & -23.40 \\
45 & AMS & 36,300 & 250 & -28.00 \\
45 & AMS & 35,180 & 180 & -31.00 \\
45 & AMS & 34,690 & 170 & -21.00 \\
47 & AMS & 42,300 & 1300 & -21.10 \\
47 & AMS & 41,100 & 800 & -21.10 \\
50 & GPC & 35,680 & 690 & -20.80 \\
51 & LSC & 22,660 & 1060 & $*$ \\
53 & AMS & 37,850 & 1100 & -21.50 \\
56 & LSC & 38,347 & 511 & -23.40 \\
57 & AMS & 38,183 & 314 & -21.01 \\
60 & AMS & 36,840 & 230 & -22.20 \\
62 & AMS & 39,340 & 580 & -21.17 \\
62 & AMS & 36,440 & 2590 & -22.23 \\
62 & AMS & 37,960 & 500 & -18.71 \\
63 & AMS & 33,230 & 300 & -22.60 \\
64 & AMS & 42,500 & 3135 & -21.50 \\
66 & LSC & $>35,300$ & $*$ & -20.96 \\
73 & AMS & 38,630 & 840 & -20.50 \\
76 & AMS & 39,600 & 350 & -21.00 \\
77 & LSC & 21,684 & 720 & -21.00 \\
78 & AMS & 33,020 & 690 & -21.00 \\
80 & LSC & $>25,400$ & & -20.00 \\
82 & AMS & $>37,200$ & $*$ & -20.20 \\
\hline
\end{tabular}

Table $2 \mathrm{~b}$ Results for Sample F.

\begin{tabular}{cllcl}
\hline Lab code & Lab type & Age $(\mathrm{yr}$ BP) & Error $(\mathrm{yr} \mathrm{BP})$ & $\delta^{13} \mathrm{C}(\%)$ \\
\hline 3 & GPC & 2585 & 15 & -21.10 \\
4 & AMS & 2485 & 35 & -20.30 \\
5 & AMS & 2540 & 35 & -20.70 \\
5 & AMS & 2500 & 45 & -24.30 \\
7 & LSC & 2450 & 45 & -20.47 \\
8 & AMS & 2490 & 30 & -20.70 \\
11 & LSC & 2450 & 80 & -20.80 \\
12 & AMS & 2765 & 35 & -20.70 \\
13 & AMS & 2500 & 35 & -26.80 \\
13 & AMS & 2620 & 40 & -18.60 \\
15 & GPC & 2440 & 25 & -21.00 \\
16 & AMS & 2577 & 36 & -20.20 \\
22 & AMS & 2509 & 29 & -20.00 \\
22 & AMS & 2530 & 31 & -19.90 \\
23 & AMS & 2500 & 32 & -20.50 \\
25 & AMS & 2520 & 20 & -20.30 \\
25 & AMS & 2530 & 20 & -20.00 \\
26 & AMS & 2581 & 25 & -20.60 \\
27 & GPC & 2570 & 30 & -20.20 \\
30 & LSC & 2865 & 62 & -20.70 \\
31 & LSC & 2740 & 40 & -16.00
\end{tabular}


Table 2b Results for Sample F. (Continued)

\begin{tabular}{lllcl}
\hline Lab code & Lab type & Age $(\mathrm{yr}$ BP) & Error (yr BP) & $\delta^{13} \mathrm{C}(\%)$ \\
\hline 37 & AMS & 2540 & 30 & -20.10 \\
37 & AMS & 2550 & 35 & -20.20 \\
38 & LSC & 3040 & 75 & -15.00 \\
39 & AMS & 2488 & 40 & -18.10 \\
40 & LSC & 2470 & 80 & -21.14 \\
41 & GPC & 4570 & 100 & -20.00 \\
43 & AMS & 2480 & 55 & -20.30 \\
43 & AMS & 2498 & 34 & -20.31 \\
44 & AMS & 2568 & 43 & -20.60 \\
44 & AMS & 2477 & 37 & -22.60 \\
45 & AMS & 2535 & 25 & -21.00 \\
45 & AMS & 2484 & 26 & -22.00 \\
47 & AMS & 2495 & 35 & -20.60 \\
47 & AMS & 2540 & 35 & -20.40 \\
50 & GPC & 2475 & 30 & -20.30 \\
51 & LSC & 2270 & 140 & $*$ \\
53 & AMS & 2540 & 30 & -19.90 \\
56 & LSC & 2545 & 30 & -22.60 \\
57 & AMS & 2517 & 44 & -20.64 \\
60 & AMS & 2475 & 25 & -20.30 \\
62 & AMS & 2485 & 25 & -21.09 \\
62 & AMS & 2405 & 35 & -21.48 \\
62 & AMS & 2550 & 30 & -19.48 \\
63 & AMS & 2640 & 45 & -14.70 \\
64 & AMS & 2480 & 35 & -20.40 \\
66 & LSC & 2555 & 35 & -20.98 \\
73 & AMS & 2480 & 40 & -20.10 \\
75 & AMS & 2414 & 39 & -21.90 \\
76 & AMS & 2537 & 18 & -27.00 \\
78 & AMS & 2435 & 60 & -20.10 \\
80 & LSC & 2970 & 70 & -20.00 \\
82 & AMS & 2690 & 100 & -19.60 \\
\hline
\end{tabular}

Table 2c Results for Sample G.

\begin{tabular}{clrll}
\hline Lab code & Lab type & Age $(\mathrm{yr}$ BP) & Error $(\mathrm{yr} \mathrm{BP})$ & $\delta^{13} \mathrm{C}(\%)$ \\
\hline 4 & AMS & 955 & 30 & -20.40 \\
5 & AMS & 1010 & 40 & -21.40 \\
5 & AMS & 930 & 50 & -22.90 \\
8 & AMS & 1080 & 25 & -19.90 \\
12 & AMS & 1385 & 30 & -21.30 \\
13 & AMS & 980 & 30 & -24.20 \\
13 & AMS & 1205 & 30 & -17.40 \\
16 & AMS & 982 & 30 & -19.80 \\
22 & AMS & 946 & 26 & -19.50 \\
22 & AMS & 962 & 28 & -19.40 \\
23 & AMS & 1007 & 42 & -20.00 \\
25 & AMS & 985 & 20 & -19.80 \\
25 & AMS & 985 & 15 & -20.00 \\
26 & AMS & 1017 & 23 & -20.20
\end{tabular}


Table 2c Results for Sample G. (Continued)

\begin{tabular}{llrll}
\hline Lab code & Lab type & Age $(\mathrm{yr}$ BP $)$ & Error $(\mathrm{yr} \mathrm{BP})$ & $\delta^{13} \mathrm{C}(\%)$ \\
\hline 37 & AMS & 940 & 40 & -19.80 \\
39 & AMS & 842 & 45 & -28.30 \\
43 & AMS & 952 & 30 & -19.91 \\
43 & AMS & 917 & 32 & -19.96 \\
44 & AMS & 1035 & 32 & -19.80 \\
45 & AMS & 980 & 24 & -21.00 \\
47 & AMS & 975 & 35 & -20.10 \\
47 & AMS & 1050 & 35 & -19.80 \\
53 & AMS & 970 & 30 & $*$ \\
55 & AMS & 975 & 45 & -21.00 \\
57 & AMS & 1104 & 42 & -19.92 \\
60 & AMS & 935 & 25 & -15.80 \\
62 & AMS & 930 & 20 & -20.88 \\
62 & AMS & 1470 & 70 & -22.07 \\
62 & AMS & 950 & 20 & -19.55 \\
63 & AMS & 980 & 40 & -19.20 \\
64 & AMS & 890 & 30 & -20.30 \\
73 & AMS & 945 & 35 & -19.80 \\
75 & AMS & 910 & 25 & -20.80 \\
76 & AMS & 1016 & 17 & -19.00 \\
78 & AMS & 900 & 60 & -19.80 \\
82 & AMS & 1110 & 90 & -21.80 \\
\hline
\end{tabular}

Table 2d Results for Sample H.

\begin{tabular}{clccc}
\hline Lab code & Lab type & Age $(\mathrm{yr} \mathrm{BP})$ & Error $(\mathrm{yr} \mathrm{BP})$ & $\delta^{13} \mathrm{C}(\%)$ \\
\hline 3 & GPC & 9540 & 25 & -17.00 \\
4 & AMS & 9510 & 35 & -16.80 \\
5 & AMS & 9540 & 40 & -15.90 \\
5 & AMS & 9530 & 50 & -15.80 \\
7 & LSC & 9480 & 70 & -16.60 \\
8 & AMS & 9365 & 50 & -16.30 \\
11 & LSC & 8770 & 120 & -16.30 \\
12 & AMS & 9725 & 65 & -16.30 \\
13 & AMS & 9290 & 50 & -17.30 \\
13 & AMS & 9560 & 60 & -17.10 \\
15 & GPC & 9580 & 45 & -16.80 \\
16 & AMS & 9518 & 43 & -16.20 \\
22 & AMS & 9545 & 40 & -15.90 \\
22 & AMS & 9690 & 45 & -16.00 \\
22 & AMS & 9573 & 40 & -15.50 \\
23 & AMS & 9500 & 70 & -16.60 \\
25 & AMS & 9525 & 20 & -16.40 \\
25 & AMS & 9555 & 25 & -16.00 \\
26 & AMS & 9592 & 38 & -16.60 \\
27 & GPC & 9485 & 45 & -16.20 \\
30 & LSC & 9581 & 67 & -17.00 \\
31 & LSC & 9510 & 60 & -11.30 \\
32 & LSC & 9250 & 100 & -15.00 \\
37 & AMS & 9490 & 40 & -16.30
\end{tabular}


Table 2d Results for Sample H. (Continued)

\begin{tabular}{|c|c|c|c|c|}
\hline Lab code & Lab type & Age (yr BP) & Error (yr BP) & $\delta^{13} \mathrm{C}(\%)$ \\
\hline 37 & AMS & 9530 & 50 & -16.30 \\
\hline 37 & AMS & 9470 & 45 & -16.30 \\
\hline 37 & AMS & 9590 & 50 & -16.30 \\
\hline 38 & LSC & 9880 & 170 & -11.20 \\
\hline 39 & AMS & 9476 & 55 & -10.10 \\
\hline 40 & LSC & 8700 & 110 & -16.75 \\
\hline 41 & GPC & 4810 & 130 & -20.00 \\
\hline 41 & GPC & 5220 & 180 & * \\
\hline 41 & GPC & 4950 & 105 & * \\
\hline 42 & GPC & 9520 & 45 & -18.10 \\
\hline 43 & AMS & 9485 & 55 & -16.36 \\
\hline 43 & AMS & 9455 & 50 & -16.22 \\
\hline 44 & AMS & 9682 & 53 & -16.10 \\
\hline 44 & AMS & 9617 & 49 & -17.20 \\
\hline 45 & AMS & 9435 & 33 & -17.00 \\
\hline 47 & AMS & 9645 & 40 & -16.60 \\
\hline 47 & AMS & 9610 & 40 & -15.90 \\
\hline 50 & GPC & 9490 & 50 & -16.20 \\
\hline 51 & LSC & 10,150 & 380 & * \\
\hline 53 & AMS & 9545 & 35 & -15.90 \\
\hline 56 & LSC & 9491 & 45 & -17.20 \\
\hline 57 & AMS & 9372 & 56 & -16.55 \\
\hline 60 & AMS & 9520 & 35 & -13.00 \\
\hline 62 & AMS & 9640 & 35 & -15.31 \\
\hline 62 & AMS & 9195 & 40 & -19.11 \\
\hline 62 & AMS & 9560 & 40 & -16.11 \\
\hline 64 & AMS & 9560 & 60 & -16.70 \\
\hline 66 & LSC & 9435 & 45 & -16.41 \\
\hline 73 & AMS & 9560 & 60 & -16.80 \\
\hline 75 & AMS & 8891 & 58 & -17.20 \\
\hline 78 & AMS & 9250 & 80 & -16.40 \\
\hline 80 & LSC & 9420 & 120 & -20.00 \\
\hline 82 & AMS & 9790 & 240 & -17.30 \\
\hline
\end{tabular}

Table 2e Results for Sample I.

\begin{tabular}{cllcl}
\hline Lab code & Lab type & Age $(\mathrm{yr}$ BP) & Error $(\mathrm{yr} \mathrm{BP})$ & $\delta^{13} \mathrm{C}(\%)$ \\
\hline 3 & GPC & 8410 & 20 & -17.00 \\
4 & AMS & 8255 & 35 & -16.60 \\
5 & AMS & 8375 & 35 & -16.40 \\
5 & AMS & 8345 & 45 & -19.50 \\
7 & LSC & 8390 & 60 & -16.70 \\
8 & AMS & 8400 & 40 & -17.10 \\
11 & LSC & 7940 & 90 & -16.60 \\
12 & AMS & 8300 & 50 & -16.70 \\
13 & AMS & 8340 & 50 & -13.60 \\
13 & AMS & 8300 & 60 & -17.50 \\
15 & GPC & 8300 & 35 & -16.50 \\
16 & AMS & 8300 & 33 & -16.40 \\
22 & AMS & 8336 & 40 & -16.20
\end{tabular}


Table 2e Results for Sample I. (Continued)

\begin{tabular}{|c|c|c|c|c|}
\hline Lab code & Lab type & Age (yr BP) & Error (yr BP) & $\delta^{13} \mathrm{C}(\%)$ \\
\hline 22 & AMS & 8380 & 40 & -16.00 \\
\hline 22 & AMS & 8451 & 36 & -15.80 \\
\hline 23 & AMS & 8320 & 47 & -16.90 \\
\hline 25 & AMS & 8335 & 20 & -16.80 \\
\hline 25 & AMS & 8355 & 25 & -17.00 \\
\hline 26 & AMS & 8357 & 36 & -17.10 \\
\hline 27 & GPC & 8355 & 40 & -16.20 \\
\hline 28 & LSC & 8370 & 240 & $*$ \\
\hline 30 & LSC & 8494 & 99 & -19.10 \\
\hline 31 & LSC & 8590 & 50 & -12.10 \\
\hline 32 & LSC & 8290 & 100 & -15.00 \\
\hline 37 & AMS & 8220 & 50 & -16.20 \\
\hline 37 & AMS & 8290 & 55 & -16.00 \\
\hline 37 & AMS & 8360 & 40 & -16.20 \\
\hline 37 & AMS & 8390 & 40 & -16.70 \\
\hline 38 & LSC & 8700 & 115 & -10.70 \\
\hline 39 & AMS & 8262 & 50 & -22.80 \\
\hline 40 & LSC & 8030 & 110 & -17.02 \\
\hline 41 & GPC & 7550 & 110 & -20.00 \\
\hline 42 & GPC & 8300 & 45 & -18.10 \\
\hline 43 & AMS & 8350 & 47 & -16.73 \\
\hline 43 & AMS & 8290 & 50 & -16.80 \\
\hline 44 & AMS & 8411 & 50 & -16.50 \\
\hline 44 & AMS & 8318 & 43 & -18.50 \\
\hline 45 & AMS & 8194 & 31 & -17.00 \\
\hline 45 & AMS & 8268 & 31 & -17.00 \\
\hline 47 & AMS & 8340 & 40 & -16.30 \\
\hline 47 & AMS & 8380 & 35 & * \\
\hline 50 & GPC & 8280 & 40 & -16.50 \\
\hline 53 & AMS & 8295 & 35 & -17.20 \\
\hline 56 & LSC & 8360 & 37 & -18.50 \\
\hline 57 & AMS & 9066 & 55 & -16.47 \\
\hline 60 & AMS & 8260 & 35 & -16.40 \\
\hline 62 & AMS & 8345 & 35 & -17.79 \\
\hline 62 & AMS & 8485 & 45 & -19.52 \\
\hline 62 & AMS & 8280 & 35 & -16.34 \\
\hline 63 & AMS & 7990 & 55 & -18.90 \\
\hline 64 & AMS & 8330 & 50 & -16.80 \\
\hline 66 & LSC & 8330 & 45 & -16.72 \\
\hline 73 & AMS & 8320 & 60 & -15.70 \\
\hline 75 & AMS & 7739 & 59 & -18.20 \\
\hline 76 & AMS & 8372 & 19 & -19.00 \\
\hline 77 & LSC & 8144 & 142 & -18.00 \\
\hline 78 & AMS & 8150 & 95 & -17.00 \\
\hline 80 & LSC & 8890 & 100 & -20.00 \\
\hline 82 & AMS & 8610 & 210 & -13.25 \\
\hline
\end{tabular}




\section{RESULTS}

In the analyses reported here, the replicate results from individual laboratories have been included as though they were an independent set of results, an assumption that is not unreasonable. Table 1 lists the laboratories that took part in the study while Table 2 presents the results as reported for samples $\mathrm{E}-\mathrm{H}$. An * indicates that a piece of information is missing. All results are given in yr BP. For Sample E, a small number of laboratories reported the age in "greater than" format. Also, the quoted uncertainty for this sample was often asymmetric, but for simplicity in format, the larger of the 2 values has been quoted. For some laboratories (6 in total), the $\delta^{13} \mathrm{C}$ was estimated, no adjustments have been made to the fractionation corrections, and the age results have not been adjusted.

Table 3 lists the summary statistics for each sample (including the mean, median, IQR [interquartile range], and range) for both ${ }^{14} \mathrm{C}$ age and $\delta^{13} \mathrm{C}$. Table 4 provides a more in-depth summary of the results for the different laboratory types.

Table 3 Detailed summary statistics for each sample.

\begin{tabular}{lrrrlrlllll}
\hline & $n$ & Mean & Median & St dev & $\mathrm{Q}_{1}$ & \multicolumn{1}{c}{$\mathrm{Q}_{3}$} & Min & Max & IQR & Range \\
\hline Sample E & & & & & & & & & & \\
$\delta^{13} \mathrm{C}$ & 57 & -21.43 & -21.00 & 2.517 & -21.60 & -20.60 & -31.80 & -15.80 & & \\
Age (BP) & 57 & 36,905 & 38,183 & 5085 & 35,320 & 40,400 & 21,684 & 42,500 & 5080 & 20,816 \\
Sample F & & & & & & & & & & \\
$\delta^{13} \mathrm{C}$ & 52 & -20.51 & -20.40 & 2.032 & -21.00 & -20.02 & -27.00 & -14.70 & & \\
Age & 52 & 2590 & 2525 & 306.6 & 2481 & 2569 & 2405 & 4570 & 88 & 2170 \\
Sample G & & & & & & & & & & \\
$\delta^{13} \mathrm{C}$ & 35 & -20.42 & -19.96 & 1.961 & -21.00 & -19.80 & -28.30 & -15.80 & & \\
Age & 35 & 1007 & 980 & 126.1 & 940 & 1017 & 842 & 1470 & 77 & 628 \\
Sample H & & & & & & & & & & \\
$\delta^{13} \mathrm{C}$ & 54 & -16.25 & -16.33 & 1.69 & -16.85 & -16.00 & -20.00 & -10.10 & & \\
Age & 55 & 9236 & 9518 & 1050 & 9435 & 9573 & 4810 & 9880 & 138 & 5070 \\
Sample I & & & & & & & & & & \\
$\delta^{13} \mathrm{C}$ & 59 & -22.57 & -22.40 & 1.556 & -23.20 & -21.86 & -29.80 & -19.00 & & \\
Age & 59 & 8325 & 8335 & 215.4 & 8280 & 8380 & 7550 & 9066 & 100 & 1516 \\
\hline
\end{tabular}

It is notable that the interquartile range, IQR $\left(\mathrm{Q}_{3}-\mathrm{Q}_{1}\right)$, is generally narrow while the standard deviation and full range show the full spread of the distribution, which can be impacted by outliers. The mean is a summary statistic that is relatively sensitive to outlying values, so the table includes the median, which is relatively robust.

Table 3 and Figure 1 highlight the presence of a number of statistical outliers and the effect they have on the results (i.e. the mean and median are different, and the range and standard deviation are large). Table 4 and Figure 1 clearly demonstrate the demographic shift, with many more AMS than radiometric results. Due to the small number of results for the GPC laboratories, the boxplots appear more stretched.

\section{Outliers}

It is clear that there are a small number of measurements or outliers that need to be screened; in a first informal analysis, these observations are marked on the boxplots. The criterion for identification (those marked by * on the boxplot) is any observation that lies more than $1.5 \times$ IQR from the median. Up to $12 \%$ of the results for any 1 sample were identified as outliers $(\sim 10 \%$ of the total 

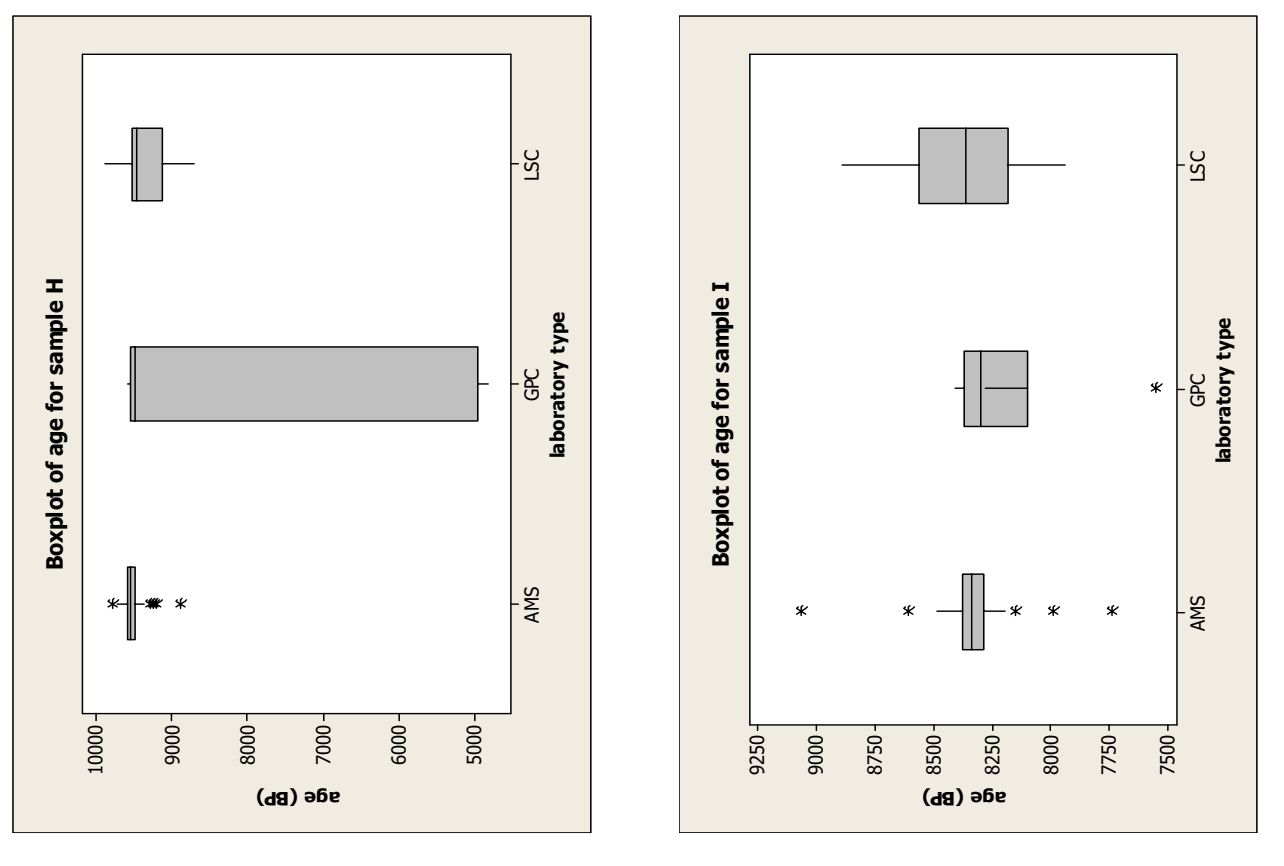

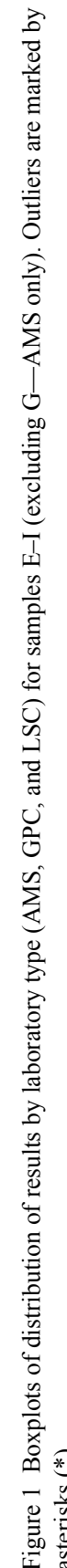
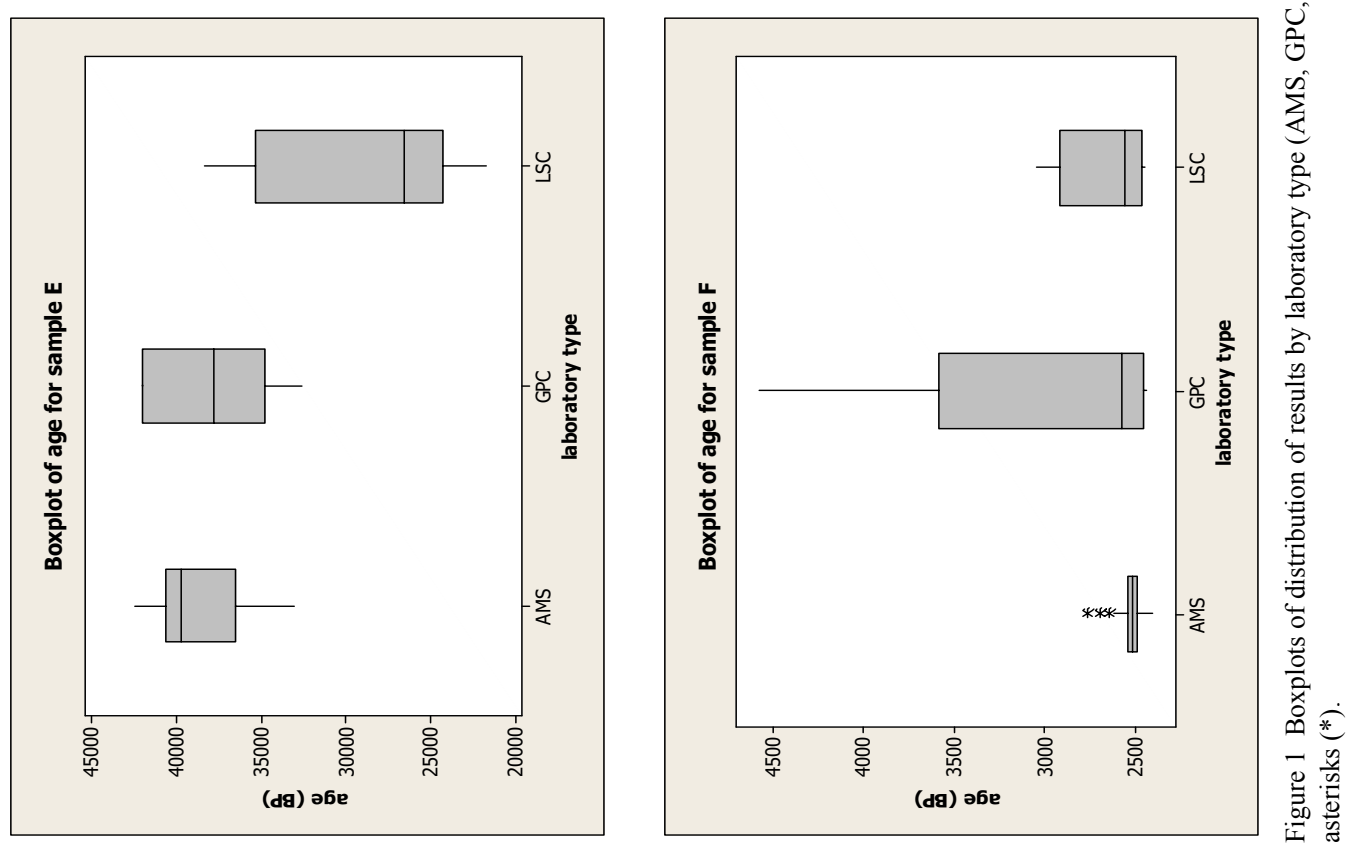
Table 4 Detailed summary statistics for each sample by laboratory type for samples E, F, H, and I.

\begin{tabular}{lrrrcrlll}
\hline & $n$ & Mean & Median & St dev & $Q_{1}$ & $Q_{3}$ & Min & Max \\
\hline Sample E & & & & & & & & \\
AMS & 40 & 38,772 & 39,695 & 2532 & 36,540 & 40,648 & 33,020 & 42,500 \\
GPC & 6 & 37,968 & 37,815 & 3928 & 34,805 & 42,015 & 32,570 & 42,060 \\
LSC & 11 & 29,537 & 26,550 & 6146 & 24,300 & 35,339 & 21,684 & 38,347 \\
Sample F & & & & & & & & \\
AMS & 38 & 2525 & 2513 & 68.6 & 24,845 & 2542 & 2405 & 2765 \\
GPC & 5 & 2928 & 2570 & 920 & 2458 & 3578 & 2440 & 4570 \\
LSC & 9 & 2676 & 2555 & 233.2 & 2460 & 2917 & 2450 & 3040 \\
Sample H & & & & & & & & \\
AMS & 38 & 9510 & 9535 & 158.4 & 9474 & 9590 & 8891 & 9790 \\
GPC & 7 & 7582 & 9485 & 2425 & 4950 & 9540 & 4810 & 9580 \\
LSC & 10 & 9352 & 9458 & 362 & 9130 & 9528 & 8700 & 9880 \\
Sample I & & & & & & & & \\
AMS & 41 & 8328 & 8335 & 176.3 & 8285 & 8373 & 7739 & 9066 \\
GPC & 6 & 8199 & 8300 & 322 & 8098 & 8369 & 7550 & 8410 \\
LSC & 12 & 8377 & 8365 & 270.1 & 8180 & 8566 & 7940 & 8890 \\
\hline
\end{tabular}

number), and these are listed in Table 5 below. When the results are summarized by laboratory type, the identified outliers change (as the distribution of results change). Interestingly, there is an increase in the relative percentage of outliers that are reported by AMS laboratories (as a direct result of the very small IQR). Omitting these measurements results in changes to the overall summary statistics and this is the basis for the preliminary consensus values shown in Table 6 .

Table 5 Identification of outliers (L, G, and A represent, respectively, LSC, GPC, and AMS).

\begin{tabular}{llllll}
\hline Sample & E & F & G & H & I \\
\hline Nr of observations & 57 & 52 & 35 & 55 & 59 \\
Nr of outliers & $6(6 \mathrm{~L})$ & $6(4 \mathrm{~L}, 1 \mathrm{G}, 1 \mathrm{~A})$ & $3(\mathrm{~A})$ & $3(3 \mathrm{G})$ & $10(5 \mathrm{~L}, 1 \mathrm{G}, 4 \mathrm{~A})$ \\
Outliers & 21684,22810, & 2740,2765, & 1205, & 4810, & 7550,7739, \\
& 24300,25400, & 2865,2970, & 1385, & 4950, & 9940,7990, \\
& 25530,26550 & 3040,4570 & 1470 & 5220 & 8030,8590, \\
& & & & & 8610,8700, \\
& & & & & 8890,9066 \\
\hline
\end{tabular}

In terms of collagen separation, most laboratories used the Longin (1971) method, with occasional modifications. There was no apparent effect of pretreatment method on the results.

There is general agreement in the results when comparing the different laboratory types (Table 6), with the possible exception of Sample E, but overall the results have also highlighted the number of individual outliers reported by the laboratories and the much-reduced IQR of results reported by AMS laboratories. Thus, the preliminary results show broad agreement across all laboratories, but there is clearly considerable scatter in the results when outliers are included. Outliers account for approximately $10 \%$ of the full set of results.

\section{Known Age}

Several of the samples had previously been dated and so there was some additional information concerning their age. For Sample G (human bone), this belonged to a 25 -yr-old female buried with a 
Table 6 Summary statistics for age with gross outliers omitted.

\begin{tabular}{lrrrrrrrr}
\hline & $n$ & Mean & Median & St dev & $Q_{1}$ & $Q_{3}$ & Min & Max \\
\hline Sample E & & & & & & & & \\
AMS & 40 & 38,772 & 39,695 & 2532 & 36,540 & 40,648 & 33,020 & 42,500 \\
GPC & 6 & 37,968 & 37,815 & 3928 & 34,805 & 42,015 & 32,570 & 42,060 \\
LSC & 5 & 35,727 & 35,339 & 1560 & 34,725 & 36,924 & 34,150 & 38,347 \\
overall & 51 & 38,379 & 39,000 & 2754 & 35,700 & 42,500 & 32,570 & 42,500 \\
Sample F & & & & & & & & \\
AMS & 37 & 2518 & 2509 & 56.5 & 2484 & 2540 & 2405 & 2690 \\
GPC & 4 & 2517 & 2522 & 71 & 2449 & 2581 & 2440 & 2585 \\
LSC & 5 & 2494 & 2470 & 51.9 & 2450 & 2550 & 2450 & 2555 \\
overall & 46 & 2516 & 2505 & 56.5 & 2480 & 2546 & 2405 & 2690 \\
Sample G & & & & & & & & \\
AMS & 33 & 974 & 975 & 58.5 & 937 & 1008 & 842 & 1110 \\
Sample H & & & & & & & & \\
AMS & 38 & 9510 & 9535 & 158.4 & 9474 & 9590 & 8891 & 9790 \\
GPC & 4 & 9524 & 9515 & 45 & 9486 & 9570 & 9485 & 9580 \\
LSC & 10 & 9352 & 9458 & 362 & 9130 & 9528 & 8700 & 9880 \\
overall & 52 & 9481 & 9523 & 213.2 & 9459 & 9578 & 8700 & 9880 \\
Sample I & & & & & & & & \\
AMS & 37 & 8326 & 8335 & 66.1 & 8290 & 8366 & 8150 & 8485 \\
GPC & 5 & 8329 & 8300 & 53.2 & 8290 & 8383 & 8280 & 8410 \\
LSC & 7 & 8340 & 8360 & 106.9 & 8290 & 8391 & 8144 & 8494 \\
overall & 49 & 8328 & 8335 & 70.4 & 8290 & 8371 & 8144 & 8494 \\
\hline
\end{tabular}

neonate in a waterlogged coffin that was dendro-dated to spring AD 1134 (5 timber-mean, one with bark edge). Previously, 6 consistent measurements from 4 laboratories gave a weighted mean of $934 \pm 12$ yr BP (A Bayliss, English Heritage, personal communication).

For Sample I (whale bone), the original laboratory entry reads: QL-1857 Jawbone of whale from sand deposits of raised beach at Svenskoya, Svalbard, Spitsbergen, Norway, dated $7950 \pm 120 \mathrm{yr}$ BP. The sample was isolated in permafrost with dates $8120 \pm 170 \mathrm{yr}$ BP above and $8350 \pm 100 \mathrm{yr} \mathrm{BP}$ below. The additional result in the records for QL-1857 is $8335 \pm 25 \mathrm{yr}$ BP. The age was calculated at $8332.5+24.0 /-23.9$ and the $\delta^{13} \mathrm{C}$ used was $-16.629 \%$. Sample $\mathrm{H}$ (whale bone) had been previously dated in Trondheim: T-13250, with a result of $9565 \pm 130 \mathrm{yr} \mathrm{BP}, \delta^{13} \mathrm{C}:-16.8 \%$. It seems clear that for samples I and $\mathrm{H}$, these new results are (after gross outlier omission) in good agreement with the earlier measurements. For Sample G, the VIRI results appear to show a difference between the mean, the median, and the previous weighted average. There is no apparent explanation for this difference.

\section{CONSENSUS VALUES}

As in previous studies, consensus values for the samples have been calculated following the procedure in Scott (2003). Most importantly, individual results are excluded from the final calculation based on two criteria: 1) their absolute value; 2) the size of the quoted error. The consensus values are then calculated as a weighted average of the remaining results and these are reported in Table 7. 
Table 7 Consensus values for VIRI Phase 2 samples.

\begin{tabular}{llcc}
\hline Sample & Nr of results used & Consensus value & Error $(1 \sigma)$ \\
\hline E & 28 & 39,305 & 121 \\
F & 44 & 2513 & 5 \\
G & 31 & 969 & 5 \\
H & 41 & 9528 & 7 \\
I & 47 & 8331 & 6 \\
\hline
\end{tabular}

\section{CONCLUSIONS}

The preliminary analysis of results from Phase 2 of VIRI has highlighted again the general and broad agreement amongst laboratories but underlines the persistent problem with outlying data values from a relatively small number of laboratories. As mentioned earlier, no corrections have been made to the results (e.g. where a fractionation correction has not been applied), nor have we, so far, as in the past, explored the reason(s) for these outlying values. Although bone is a less commonly dated material, reflected in the smaller number of participants, the scatter of results is broadly comparable to that observed in earlier intercomparisons for other materials of roughly similar age (e.g. samples B and D in Phase 1). Given the interest in pretreatment effects, we looked at this factor and observed no statistically significant difference between methods, although a relatively small number of laboratories used methods other than Longin (1971).

The same demographic shift to more AMS and fewer radiometric laboratories is apparent from the list of participating laboratories. Overall, the number of participating laboratories is slightly lower than in Phase 1, but still represent a very healthy participation rate.

\section{ACKNOWLEDGMENTS}

The authors gratefully acknowledge the financial support of English Heritage. Samples for Phase 2 were provided by Steinar Gulliksen, Paula Reimer, Tom Higham, Alex Bayliss, and Ganna Zaitseva. The authors are also grateful to all participating laboratories.

\section{REFERENCES}

Longin R. 1971. New method of collagen extraction for radiocarbon dating. Nature 230(5291):241-2.

Scott EM 2003. The Third International Radiocarbon Intercomparison (TIRI) and the Fourth International Radiocarbon Intercomparison (FIRI). Radiocarbon 45: (2):135-408.
Scott EM, Cook GT, Naysmith P, Bryant C, O’Donnell D 2007. A report on Phase 1 of the 5th International Radiocarbon Intercomparison (VIRI). Radiocarbon 49(2):409-26. 